\title{
Philosophiques
}

\section{Jacob T. Levy, Rationalism, Pluralism, and Freedom, Oxford, Oxford University Press, 2015}

\section{François Boucher}

Volume 45, numéro 1, printemps 2018

URI : https://id.erudit.org/iderudit/1048635ar

DOI : https://doi.org/10.7202/1048635ar

Aller au sommaire du numéro

Éditeur(s)

Société de philosophie du Québec

ISSN

0316-2923 (imprimé)

1492-1391 (numérique)

Découvrir la revue

Citer ce compte rendu

Boucher, F. (2018). Compte rendu de [Jacob T. Levy, Rationalism, Pluralism, and Freedom, Oxford, Oxford University Press, 2015]. Philosophiques, 45(1),

328-333. https://doi.org/10.7202/1048635ar

Ce document est protégé par la loi sur le droit d'auteur. L'utilisation des services d'Érudit (y compris la reproduction) est assujettie à sa politique d'utilisation que vous pouvez consulter en ligne.

https://apropos.erudit.org/fr/usagers/politique-dutilisation/ 
différenciation n'est effectuée entre logos et muthos ${ }^{14}$. Deuxièmement, en raison d'une conception du temps et d'une conception de la vérité qui semblent invalider la possibilité d'une «histoire». La "philosophie» indienne, explique Hulin, se fonde en effet sur une conception cyclique du temps, dans laquelle la vérité est d'emblée donnée au groupe des Brahmanes, vérité dont la transmission est progressivement dégradée avant d'être régulièrement retrouvée. L'idée selon laquelle le savoir est "laborieusement acquis par l'homme » — qui semble en retour constituer l'une des conditions d'une histoire de la philosophie - est totalement absente de ce contexte, dans lequel la notion d'originalité ne peut donc pas non plus avoir de sens.

Nicolas Zufferey insiste quant à lui sur les précautions épistémologiques qui s'imposent à celui qui s'intéresse à la philosophie chinoise, précautions qui, si elles sont ignorées, risquent de conduire l'interprète à conclure hâtivement à l'incohérence des auteurs. En s'appuyant sur sa lecture de Wang Chong, Zufferey souligne lui aussi qu'il faut considérer la conception singulière de la vérité que déploie la pensée chinoise: la vérité n'est pas conçue comme un absolu, indépendant des circonstances; la vérité est à adapter au contexte. Il ajoute qu'il faut mesurer l'importance du statut particulier du penseur chinois, qui est toujours en même temps un conseiller du pouvoir politique. Selon Zufferey, ce n'est qu'en étant sensible à ces éléments de contexte qu'on parvient à retrouver la cohérence du texte et à dépasser les contradictions apparentes.

Finalement, en donnant à voir une diversité d'approches et de pistes, ce volume constitue une contribution essentielle qui alimente et nourrit la réflexion en épistémologie de l'histoire de la philosophie.

OPHÉLIE DESMONS

ESPE de Paris,

Université Paris-Sorbonne

\section{Jacob T. Levy, Rationalism, Pluralism, and Freedom, Oxford, Oxford University Press, 2015.}

Les personnes libres s'associent pour poursuivre les finalités qu'elles partagent. Elles forment des associations telles que des Églises, afin de pratiquer et promouvoir conjointement une religion, ou des universités, pour approfondir et propager les connaissances. Les individus qui partagent une langue ou une origine ethnique se regroupent afin d'exercer leur autonomie politique ou de faire valoir leurs revendications culturelles. Ces groupes sont des associations intermédiaires, ils s'interposent entre l'État et l'individu, jouent

14. Michel Hulin, «La "philosophie" indienne a-t-elle une histoire?», dans Vincent Citot, dir., Problèmes épistémologiques en histoire de la philosophie, p. 298. 
un rôle central dans la socialisation des personnes, et leur donnent une voix dans la sphère publique.

Dans son ouvrage Rationalism, Pluralism, and Freedom (Oxford University Press, 20I 5), Jacob T. Levy examine les enjeux politiques qui naissent de la relation triadique entre les personnes, les États et les groupes ou associations intermédiaires. En plus des Églises, des universités et des groupes culturels, les ordres monastiques, les parlements régionaux et les États fédérés, les villes et les guildes médiévales composent les associations intermédiaires qui sont étudiées dans ce livre. Pour Levy, ces associations ont des engagements éthiques profonds et partagés par leurs membres, c'est-à-dire, une mission ou un but qui leur est propre, comme la propagation d'une foi ou le maintien d'un mode de vie. Elles ont aussi une structure juridique plus ou moins formelle qui institue une forme d'autorité. Les groupes fortement institutionnalisés, comme l'Église catholique, et les groupes informels, comme les groupes culturels, partagent ces deux caractéristiques (p. I6-I7). Toutefois, ce livre exclut délibérément de son champ d'études les partis politiques et les lobbys, qui représentent les intérêts de groupes particuliers face à l'État, ainsi que les corporations commerciales et les unions syndicales, lesquelles représentent des intérêts particuliers au sein du marché. Les associations qui sont examinées par Levy sont celles qui sont fondamentalement préoccupées par des questions de gouvernance interne et affirment une conception éthique substantielle, ce qui n'est pas le cas des partis politiques, des firmes et des syndicats (p. I3).

Levy s'intéresse tout particulièrement aux tensions que crée la relation triadique individu - association - État pour les penseurs du libéralisme. D'une part, la vie de groupe découle de la liberté individuelle. Elle peut être l'expression du choix d'une conception de la vie bonne et une manière de résister aux tendances centralisatrices et majoritairistes de l'État moderne. La tyrannie de la majorité, l'anticléricalisme et la suspicion envers les groupes intermédiaires sont des figures récurrentes de l'excès de concentration de pouvoir étatique au détriment de la liberté associative. D'autre part, les groupes intermédiaires peuvent être des sources d'oppression interne en instaurant des relations hiérarchiques entre leurs membres et en excluant les non-membres. C'est le problème bien connu en philosophie politique des minorités à l'intérieur des minorités. Par ailleurs, les dérives centralisatrices de l'État sont bien souvent le résultat de la promotion de la liberté individuelle par l'État qui se méfie excessivement du pouvoir des groupes intermédiaires et interfère avec des formes légitimes d'association (p. 34).

La thèse centrale du livre est que le libéralisme est traversé par un affrontement entre deux courants: le rationalisme et le pluralisme. Le premier est associé à la théorie du contrat social, à l'autonomie rationnelle, au constitutionnalisme moderne, et à l'uniformité des normes régissant la vie des citoyens. Le rationalisme privilégie le pouvoir de l'État en mettant l'accent sur les dangers que posent les groupes intermédiaires pour la liberté 
individuelle, et le besoin de protéger l'individu des traditions religieuses et culturelles oppressantes ainsi que des élites locales. Le second est associé à la tolérance, à la diversité culturelle, au pluralisme juridique et au constitutionnalisme ancien. Le pluralisme est par nature inquiet de la concentration du pouvoir et voit l'État moderne comme intolérant, homogénéisant et en proie aux dérives centralisatrices qui minent la diversité (p. 27-30). Cette caractérisation du libéralisme est intéressante et originale puisqu'elle révèle une véritable tension qui est souvent occultée par d'autres lectures du libéralisme. On présente en effet généralement ce courant de pensée comme étant plutôt traversé par un désaccord au sujet des valeurs qui le fondent, opposant ainsi le libéralisme compressif d'inspiration millienne fondé sur un idéal d'autonomie individuelle, qu'on retrouve entre autres dans les travaux de Joseph Raz et de Will Kymlicka ${ }^{1}$, au libéralisme politique d'inspiration kantienne fondé sur la valeur de la tolérance et mis de l'avant chez Rawls et Charles Larmore ${ }^{2}$, par exemple.

Selon Levy, le désaccord entre pluralistes et rationalistes n'a rien à voir avec les principes moraux premiers (la tolérance contre l'autonomie, par exemple). Il s'agit plutôt d'un désaccord entre deux manières de comprendre le monde social et politique (p. II, 57) et de concevoir les dynamiques de pouvoir impliquées par le triangle individu-association-État. Entre l'État et les groupes intermédiaires, quel pouvoir constitue la plus grande source de domination? Telle est la question fondamentale de la pensée libérale. Levy étudie celle-ci en évitant d'idéaliser l'État et les groupes intermédiaires, et en voyant l'un et les autres "comme étant similairement enclins aux comportements non angéliques» (p. 60). Il propose non pas une théorie pure de la justice idéale qui prescrit ce qui doit être fait dans une société parfaitement juste, mais plutôt une théorie politique impure et non idéale qui s'appuie sur des observations sociologiques et historiques à propos du pouvoir exercé par les États et les groupes intermédiaires.

Le chapitre III, dont les thèses sont revisitées au chapitre X, est central à l'ouvrage. Il développe un cadre d'analyse des dynamiques de pouvoir propres au triangle individu-groupe-État. Levy y révèle les logiques et les mécanismes qui mènent aux excès de pouvoir associatif ou étatique. Il s'appuie sur le constat que l'autorité des groupes intermédiaires génère du pouvoir. Ce pouvoir peut être totalisant et ne laisser aucune porte de sortie effective et, comme tout pouvoir, il peut être exercé de manière arbitraire (p. 47-50). Cela fonde la méfiance de l'État moderne à l'égard des associa-

1. Joseph Raz, The Morality of Freedom, Oxford, Oxford University Press, I986; Will Kymlicka, La citoyenneté multiculturelle. Une théorie libérale du droit des minorités, trad. P. Savidan, Paris, La Découverte, $200 \mathrm{I}$.

2. John Rawls, Libéralisme politique, trad. C. Audart, Paris, Presses Universitaires de France, 2006; Charles Larmore, "Political Liberalism", Political Theory, I990, I8/3, 339360. 
tions intermédiaires. Nourri par cette méfiance, l'État a eu tendance à exproprier les ressources des associations et à brimer leur secret et leur vie privée (p. 6I-63). De plus, les administrateurs de l'État, en raison d'impératifs bureaucratiques, ont tendance à se conformer à la figure de l'homme du système (Smith) ou à l'esprit de l'uniformité (Montesquieu) : il est plus facile, plus efficace, de gouverner une population lorsque celle-ci opère selon des standards communs (langue, unité de mesure, règlements). Ce point de vue bureaucratique tend à négliger et supprimer la diversité (p. 66-68, I75-I77). La vie de groupe produit également ses propres dérives. En raison de leur caractère intergénérationnel, les groupes limitent la liberté individuelle: «Les associations libres pour une génération deviennent héritées pour la génération suivante» (p. 7I). De plus, en poursuivant en commun des buts partagés, les individus acceptent de s'assujettir à un pouvoir associationnel (p. 7I-73). Or ceux qui exercent une telle autorité sont d'autant plus en position d'abuser de cette position que l'État s'abstient d'intervenir dans la vie associative afin de respecter les exigences du pluralisme. Par ailleurs, les groupes ont intérêt, pour se faire reconnaitre et attirer des membres, à se distinguer des autres groupes et de la société dans son ensemble. Pour ce faire, ils ont tendance à se radicaliser, à adopter des normes de fonctionnement internes moins progressistes et en contradiction avec les exigences de la société libérale (p. 75-8I).

Levy défend deux thèses dérivées de sa thèse centrale (laquelle affirme que le libéralisme est traversé par un affrontement entre rationalisme et pluralisme), l'une historique, l'autre normative. La thèse historique affirme que la tension entre rationalisme et pluralisme traverse toute l'histoire de la pensée libérale. Cette tension n'est pas une anomalie, bien que le libéralisme contemporain ait tendance à s'associer exclusivement au courant rationaliste et à discréditer ses origines pluralistes. La thèse normative affirme que cette tension est irréductible et qu'il serait mal avisé de vouloir la résoudre en proposant de systématiquement subordonner les associations intermédiaires à l'État, ou vice-versa. Bien qu'il ne cache pas son penchant pluraliste, Levy reconnaît la nécessité de prendre en considération les préoccupations rationalistes, qui doivent parfois l'emporter.

Levy défend cette thèse normative dans les trois premiers chapitres du livre et dans les deux derniers. Cette thèse est une négation de la thèse de la congruence, laquelle affirme que la vie interne des groupes intermédiaires doit reposer sur les mêmes normes qui régissent l'État libéral. Selon la thèse de la congruence, pour éviter de dominer leurs membres, les associations se doivent d'être des mini-États démocratiques (p. 5I-52). Mais Levy rejette la congruence en soulignant que les associations sont des organisations d'une nature fondamentalement différente des États puisque leur raison d'être est de faire la promotion de finalités substantielles, d'une conception particulière du bien, alors que l'État libéral doit éviter de faire siennes de telles finalités. S'il ne fait pas sens de demander aux groupes de ne pas traiter leurs 
membres différemment des non-membres (par exemple, demander aux Églises d'accepter les non-croyants), l'État doit quant à lui être impartial (ne pas discriminer entre croyants et non-croyants) (p. 53). Les associations intermédiaires ne sont pas des États, et ces deux types d'organisations doivent opérer selon des standards différents. Cela vaut également dans le cas des associations complexes, celles qui sont pluralistes et contiennent une sphère associationnelle interne (p. 266-267). Levy illustre ce point au chapitre XI en discutant de la liberté académique et des relations entre État, universités et associations universitaires. Il conclut l'ouvrage en critiquant les tentatives de réconcilier rationalisme et pluralisme de trois grands penseurs: Hegel, Rawls et Taylor. Pour Levy, si nous nous soucions de la liberté, nous devons admettre une part inévitable de confrontation et de disharmonie dans nos vies sociales et nos théories politiques (p. 295).

Levy défend la thèse historique par une étude approfondie des débats entre pluralistes et rationalistes depuis l'époque médiévale. Puisque la pensée libérale contemporaine s'associe au rationalisme et occulte ses origines pluralistes, Levy cherche à dévoiler ces origines. Il situe les racines du pluralisme dans la pensée politique médiévale des $\mathrm{XI}^{\mathrm{e}}$ et $\mathrm{XII}^{\mathrm{e}}$ siècles, notamment dans le constitutionnalisme ancien (germanique ou gothique), une forme de pluralisme juridique qui applique un régime juridique différencié selon l'appartenance ethnoculturelle des personnes, dans l'ordre féodal dans lequel la souveraineté est fortement éclatée et dans l'émergence des ordres monastiques, des guildes et des villes médiévales (chapitres IV et V). Levy montre ensuite comment pluralistes et rationalistes ont été en dialogue à différentes périodes. Ainsi, au chapitre VI, il oppose Montesquieu, figure centrale du pluralisme, à Voltaire le rationaliste. Il fait dialoguer Tocqueville et Mill au chapitre VIII, le premier ayant souligné le rôle des associations intermédiaires dans la jeune démocratie américaine alors que le second soutenait que le despotisme local est plus dangereux que la centralisation de l'État. Au chapitre IX, il montre comment les pluralistes britanniques du début du $\mathrm{XX}^{\mathrm{e}}$ siècle (Figgis, Cole et Laski) se sont opposés à Lord Acton, qui voyait dans le constitutionnalisme ancien une menace au progrès de la liberté. Au chapitre VII, il examine les débats entre rationalistes et pluralistes dans la foulée des révolutions française (Tracy, Constant, Burke, Paine) et américaine (Franklin, Jefferson, Adams, Madison et la société de Cincinnati).

L'étude des sources historiques du pluralisme et du rationalisme faite par Levy est riche et a une valeur en soi. Elle lui permet de démontrer de manière convaincante la thèse de la persistance et de la longévité de ce clivage interne à la pensée libérale. Néanmoins, bien qu'elle illustre brillamment une position importante dans la pensée libérale, la démonstration de Levy visant à soutenir la thèse normative de l'irréductibilité pose quelques problèmes. L'argument principal de la thèse de l'irréductibilité repose sur la distinction entre l'État et les associations intermédiaires. Il me semble que cette distinction tient effectivement la route pour les associations volontaires 
de nature religieuse, académique ou culturelle. Mais Levy inclut également dans son concept d'associations intermédiaires les États fédérés, les parlements régionaux et les villes. Or, si ces organisations partagent avec les associations volontaires une préoccupation par rapport aux risques de centralisation et de dérives majoritaires en plus d'être l'objet des suspicions des États centraux (pensons aux États fédérés qui constituent des nations minoritaires), il n'en reste pas moins qu'il s'agit de paliers de gouvernement qui imposent leurs lois à tous les résidents d'un territoire donné, et non pas d'associations volontaires poursuivant des finalités substantielles définies par une conception de la vie bonne. Levy reconnaît qu'il y a des différences entre gouvernements subétatiques et associations volontaires, mais argue néanmoins en faveur d'un traitement commun (p. I5-I6). Cette différence est toutefois cruciale pour la thèse de la congruence. Si l'argument anticongruence de Levy est convaincant à l'égard des Églises et des universités, il ne l'est pas en ce qui concerne les gouvernements subétatiques. En effet, ceux-ci sont, précisément, des gouvernements. Ce sont des organisations qui doivent, d'un point de vue libéral, s'abstenir de poursuivre une conception de la vie bonne et sont censées faire respecter l'égale liberté de tous ceux et celles qui habitent leur territoire. Si l'on peut tolérer que l'Église catholique s'écarte des valeurs démocratiques en refusant la prêtrise aux femmes et en excluant les non-croyants, on ne saurait donner une telle marge de manœuvre aux provinces et aux villes (afin de leur permettre, par exemple, d'exclure les femmes des postes de direction). D'un autre côté, exiger des gouvernements subétatiques qu'ils se conforment aux principes fondamentaux du libéralisme ne revient pas à nier toute forme de diversité. Sans brimer ces principes libéraux fondamentaux, un État fédéré peut se doter d'une langue officielle distincte de la langue de la majorité, et une ville peut adopter un taux de taxation plus élevé que les villes voisines afin de soutenir une vaste infrastructure de transport en commun. La question de la congruence se pose donc différemment pour les gouvernements subétatiques et pour les associations volontaires, différence que néglige Levy. Ainsi, si ce livre offre une discussion minutieuse et érudite des sources historiques de la liberté associationnelle et redonne ses lettres de noblesse au pluralisme libéral tout en adoptant une perspective non idéale en théorie politique, il ne tient cependant pas suffisamment compte des différences entre diverses catégories de groupes intermédiaires. Il s'agit néanmoins d'une contribution importante et d'un outil formidable qui enrichit notre compréhension de la complexité du libéralisme.

FRANÇOIS BOUCHER 